\section{OSx86 for the Masses?}

Apple's announcement in June that it would begin using Intel chips took the computing world by surprise. The design-savvy company opted to begin using Intel chips in 2006, mostly because of Intel's expertise in manufacturing low-power chips. Participants in the Apple Developer Connection recently received a developer's kit that allows them to test their OS X code on Macs fitted with Intel chips, now increasingly known as "MacIntels." The kit was enabled with security features to prevent others from installing OS X on non-Macintosh $\mathrm{x} 86$ boxes.

Not surprisingly, hackers cracked the security protections-which explains why Web sites have sprung up showing videos of Dell laptops booting OS X, or "OSx86," as some are calling it. Apple repeatedly claimed the new version of OS $\mathrm{X}$ is designed to work only with Apple hardware, but the question now is whether Apple would ever consider selling its flagship OS to owners of generic, Intelor AMD-powered white-box computers. Such a move would cut into Mac sales, but isn't it possible that selling the heralded Mac OS to the PC majority might more than make up for the losses? If Apple decides to go that route, we'll begin to see an OS battlefield that's far less hardware-dependent. And if the trend were to continue? Then we could be faced with the most unlikely of hardware/OS combinations_-gasp!-Win XP running on a Mac. WANT MORE?

http://www.computerworld.com/softwaretopics/os/ macos/story/0,10801,103928,00.html

\section{OS? We Don't Need No Stinkin' OS}

While some companies battle it out in the OS arena, others choose to direct their efforts elsewhere. Take Google, whose growing number of offerings is challenging Microsoft's and Apple's, sans OS.

Even without a homegrown operating system on which to launch their products, Google and others such as Yahoo are taking an approach similar to that of their rivals to promote their adoption. The key? Open the software to developers. The majority of Google's software has published APIs that developers can leverage to create new, custom-built products and services. The strategy has really taken off. For example, a quick survey of socalled "mashup" sites using the Google Maps API reveals

\section{Taking a \\ second look AT \\ THE NEWS SO YOU \\ DON'T HAVE TO}

everything from a dating site that shows roughly where a potential date lives (stalkers take note: it drills down only as far as the Zip code), to a site that shows where the worst potholes are in New York City, to one that shows the locations of the latest crimes committed in Chicago. What makes these offerings stand out is that because the services are accessed via the Web, users and developers are not dependent on a particular operating system for access. With a similar, service-oriented strategy taking hold in the enterprise world (see Salesforce.com), the lesson might be that the much-hyped OS wars ultimately don't matter.

WANT MORE?

http://news.com.com/2100-1007_3-5843402.html

\section{Real Crime Descends upon Virtual World}

If the Web is the ultimate platform, then the MMO (massive multiplayer online) gaming universe is its ultimate playground. Games such as EverQuest II are so sophisticated, and their immersion experience so deep, that it's no wonder why many spend hours every day engrossed in their online virtual worlds. But time is not all they spend. EverQuest and others such as World of Warcraft have evolved elaborate virtual economies that closely mimic the workings of the real world.

But sometimes the virtual world mimics aspects of our real world we would rather do without. Two recent incidents illuminate this unfortunate turn. In the first, hackers developed a way to duplicate valuable virtual goods, sending their value within the game's economy plummeting and inflating its virtual currency 20 percent. The second incident saw characters from the roleplaying game Lineage "mugged" for their goods by seemingly invincible bots set loose into the gaming space by real, flesh-and-blood criminals. The trend here can be summed up by security expert and Queue contributor Bruce Schneier: "[E]very form of theft and fraud in the real world will eventually be duplicated in cyberspace." WANT MORE?

http://www.newscientist.com/article.ns?id=dn7865 http://www.newscientist.com/article.ns?id=dn7846 Q 